\title{
Utilidad de la alfa-fetoproteína en el diagnóstico prenatal de defectos del tubo neural y anomalías cromosómicas.
}

\section{Artículo Original}

Pilar Salas-Chaves ${ }^{1}$, Sara Rodríguez-Aguilar², Lowella Cunningham-Lucas² ${ }^{2}$ Isabel Castro-Volio ${ }^{1}$.

${ }^{1}$ Instituto de Investigaciones en Salud (INISA), Universidad de Costa Rica, ${ }^{2}$ Instituto Costarricense de Investigación y Enseñanza en Nutrición y Salud, San José, Costa Rica.

\section{RESUMEN.}

Introducción. Este es un estudio retrospectivo que señala la utilidad de la determinación de la alfafetoproteína (AFP), en el líquido amniótico de 96 mujeres embarazadas costarricenses captadas en la Unidad de Perinatología del Hospital R.A. Calderón Guardia (Ciudad de San José, Costa Rica) entre las semanas 15 y 25 de gestación entre los años 1995 y 1996, para realizarse diagnóstico prenatal por amniocentesis. Este procedimiento se realizó en todas aquellas pacientes identificadas como de alto riesgo genético con el objetivo de detectar cromosomopatía fetal con la finalidad de brindar adecuada atención obstétrica y pediátrica además de asesoramiento genético.

Material y Métodos. Los procedimientos de cultivo celular, análisis cromosómico y la determinación de los niveles de alfa-fetoproteína ya han sido descritos anteriormente. Los valores de la concentración de AFP se expresaron como múltiplos de la mediana (MM), considerándose límite normal superior 2.5 MM y límite inferior $0.25 \mathrm{MM}$.
Resultados. Los límites de confiabilidad en que se encontraron los valores de la mediana fueron 562$1196 \mathrm{KU} / \mathrm{l}$ mientras que el límite normal superior fue de 1404-2989 KU/l. Cuatro casos tuvieron valores de AFP superiores a la mediana correspondiendo citogenéticamente a: Síndrome de Turner, encefalocele y dos fueron cariotipos normales. Otros cuatro casos tuvieron resultados inferiores a $0.25 \mathrm{MM}$ observándose un caso de trisomía 21, trisomía 18, onfalocele y un cariotipo normal.

Discusión. El diagnóstico prenatal de cromosomopatía permitió el asesoramiento genético y el manejo obstétrico y pediátrico de manera adecuada. (Rev Biomed 2003; 14:5-10)

Palabras clave: alfa-fetoproteína, amniocentesis, defectos del tubo neural, diagnóstico prenatal, cromosomas humanos

Solicitud de sobretiros: Dra. Pilar Salas-Chaves, Instituto de Investigaciones en Salud (INISA), Ciudad Universitaria "Rodrigo Facio", Universidad de Costa Rica, San José, Costa Rica. Teléfonos: 224-36-68, Fax: 207-51-30, Dirección electrónica: msalasc@cariari.ucr.ac.cr Recibido el 9/Noviembre/2001. Aceptado para publicación el 12/Julio/2002.

Este artículo está disponible en http://www.uady.mx/ biomedic/rb031413.pdf

Vol. 14/No. 1/Enero-Marzo, 2003 
P Salas-Chaves, S Rodríguez-Aguilar, L Cunningham-Lucas, I Castro-Volio.

\section{SUMMARY.}

Utility of the alpha-fetoprotein in the prenatal diagnosis of neural tube defects and chromosomal abnormalities.

Introduction. The present study focuses on the utility of AFP in prenatal diagnosis of 96 Costa Rican pregnant women.

Material and methods. In these women, genetic amniocentesis and AFP levels were done between the $15^{\text {th }}$ and $25^{\text {th }}$ week of gestation. There were two main reasons for referral: abnormal ultrasound assessment and advanced maternal age.

Results. The concentration values of AFP were expressed as multiples of the median, considering a value of $2.5 \mathrm{MoM}$ as a normal superior limit and of 0.25 as the inferior limit. The confidence limits of the median were $562-1196 \mathrm{KU} / \mathrm{l}$ and the $2.5 \mathrm{MoM}$ value was located between 1404-2989 KU/l. Four cases had AFP levels higher than the median: one case of Turner Syndrome, another case of encephalocele and two normal karyotypes. Four other cases had AFP levels lower than $0.25 \mathrm{MoM}$ : a case of trisomy 21 , a case of trisomy 18, a case of omphalocele and a normal karyotype.

Discussion. Prenatal diagnosis of fetal defects allowed genetic counseling as well as better obstetric management and pediatric care.

(Rev Biomed 2003; 14:5-10)

Key words: alpha-fetoprotein, amniocentesis, neural tube defects, prenatal diagnosis

\section{INTRODUCCIÓN.}

Actualmente es práctica común en los países con mayor desarrollo de los servicios asistenciales de genética, el tamizaje de defectos del tubo neural (DTN) y otras malformaciones congénitas, gracias a las cuantificaciones de alfa-fetoproteína (AFP) tanto del suero materno (de toda la población gestante en general), como del líquido amniótico de las embarazadas con alto riesgo de malformación fetal ya sea por antecedentes de defectos del tubo neural en un producto previo o en uno de los padres, o por añosidad materna, etc. Estas políticas han tenido un enorme impacto en la prevalencia al nacer de los DTN y de otros defectos estructurales fetales que de igual manera contribuyen con cantidades anormales de AFP al líquido amniótico y a la circulación materna (1).

La AFP es una glicoproteína con peso molecular de 70000 dalton, la cual es sintetizada por el embrión en un inicio en el saco vitelino y luego en el tracto gastrointestinal y el hígado, a una tasa que alcanza los $30 \mathrm{mg}$ diarios en el segundo trimestre. El feto produce grandes cantidades de AFP que es secretada a la circulación fetal, esta proteína pasa el líquido amniótico a través de la orina y después de la duodécima semana de gestación, por vías aún no definidas, a la circulación materna (2).

La relación existente entre los niveles altos de AFP y los defectos del tubo neural, se sospechó 30 años atrás, cuando Brock y colaboradores en 1972, informaron de anencefalia y espina bífida con niveles altos de AFP antes y después de la semana 24 de gestación (3).

Bajo el nombre de defectos del tubo neural (DTN) se agrupan una serie de anomalías congénitas, como anencefalia, espina bífida y encefalocele. Este grupo de defectos congénitos se halla entre los más comunes, alcanzando niveles de incidencia altos en algunas poblaciones (4).

La etiología de los DTN involucra factores ambientales y genéticos. En el caso de los factores ambientales, se ha identificado la utilización del ácido fólico como un factor protector contra los DTN, por el contrario el abuso del alcohol en el primer mes de embarazo se asocia con los DTN (5). Estudios epidemiológicos han demostrado variaciones geográficas, temporales, en el nivel socioeconómico, en la dieta materna y en la exposición a drogas en la incidencia de los DTN.

Dada la disponibilidad de reactivos comerciales para determinar los niveles de AFP en suero materno, en los años 80 se iniciaron programas de tamizaje de los niveles de AFP en los Estados Unidos. Posteriormente en 1984, Merkatz y Aickle, encontraron la asociación de niveles bajos de AFP y defectos cromosómicos (6).

\section{Revista Biomédica}




\section{Alfa-fetoproteína en el diagnóstico prenatal.}

El objetivo de este estudio fue describir la utilidad diagnóstica de la concentración de AFP en líquido amniótico de embarazadas costarricenses y correlacionar los niveles altos y bajos de AFP con los DTN y otras anomalías cromosómicas.

\section{MATERIALES Y MÉTODOS.}

Se determinaron retrospectivamente los niveles de AFP en 96 líquidos amnióticos de 96 mujeres embarazadas con edades gestacionales de 15 a 25 semanas, que fueron atendidas en la Unidad de Perinatología del Hospital R.A.Calderón Guardia (Ciudad de San José, Costa Rica) entre 1995 y 1996, para realizarse un diagnóstico prenatal por amniocentesis previo consentimiento informado de la paciente.

La amniocentesis se realizó en todas aquellas pacientes identificadas como de alto riesgo genético (edad materna igual o superior a 35 años, ella o el padre del feto era portador de una translocación o inversión cromosómica, antecedente de defecto cromosómico o feto polimalformado en un embarazo anterior y ultrasonograma anormal). Las punciones fueron transabdominales, guiadas por ultrasonografía (7) y tenía como objetivo identificar cromosomopatía fetal en voluntarias con embarazos de alto riesgo genético a fin de brindar adecuada atención obstétrica, pediátrica y asesoramiento genético.

El cultivo celular y análisis cromosómico ya han sido descritos $(7,8)$ así como los procedimientos para la determinación de los niveles de AFP $(9,10)$.

Se compararon los valores obtenidos con los resultados del diagnóstico prenatal de cromosomopatía, haciendo énfasis en aquellos casos cuyos resultados estuvieron por encima o debajo de los múltiplos de la mediana (MM) 2.5 y 0.25 respectivamente. Los parámetros de control de calidad interna de los ensayos realizados, tanto de la variabilidad intra-e interensayo de los sueros control como de la unión máxima y la unión no específica, mostraron seradecuados según las recomendaciones del fabricante (11).

El análisis estadístico de la información se realizó con el programa Excel de Microsoft Office 98 y el programa PC/RIA Versión 2 del Organismo
Internacional de Energía Atómica (12).

\section{RESULTADOS.}

Los niveles de AFP durante la gestación pueden observarse en la figura 1. La línea central se ha obtenido uniendo los valores de la mediana de cada una de las semanas estudiadas. Las líneas superior e inferior se han obtenido de forma semejante para los MM $2.5 \mathrm{y}$ 0.25 , respectivamente.

Debido al interés clínico que tiene detectar a las pacientes que tienen riesgo de ser portadoras de un feto con DTN, en el cuadro 1 se muestran los valores de la mediana al determinar la AFP en el líquido amniótico entre las semanas 15 y 25, así como sus múltiplos. Los límites de confiabilidad en que se encontraron los valores de la mediana fueron de 5621196 mientras que el múltiplo de 2.5 se situó entre 1404-2989.

Los resultados fueron clasificados como normales, elevados y bajos, en función de si se hallaban entre los MM 2.5-0.25, superaron estas cifras o no las alcanzaron, respectivamente. Los 4 casos que tuvieron valores superiores al valor de la mediana pero no sobrepasaron $2.5 \mathrm{MM}$ correspondieron citogenéticamente a: Síndrome de Turner, encefalocele (defecto cerrado) y dos cariotipos normales. Cuatro resultados no alcanzaron 0.25MM observándose un caso de trisomía 21, trisomía 18 , onfalocele y un cariotipo normal. Un

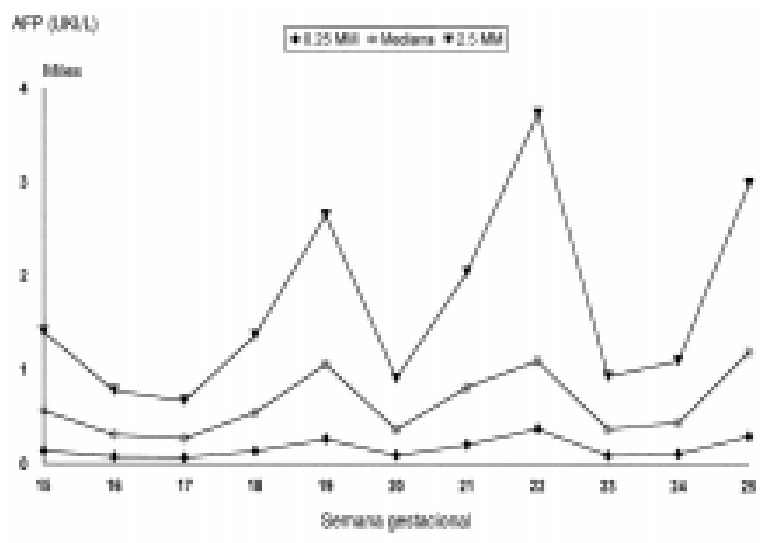

Figura 1.- Niveles normales de alfafetoproteína (AFP), en líquido amniótico de mujeres embarazadas con productos sanos, según semana gestacional, Costa Rica, 1995-1996. $\mathrm{MM}=$ Múltiplos de la mediana

Vol. 14/No. 1/Enero-Marzo, 2003 
P Salas-Chaves, S Rodríguez-Aguilar, L Cunningham-Lucas, I Castro-Volio.

Cuadro 1

Niveles de Alfa-fetoproteína (AFP) en el líquido amniótico de mujeres embarazadas con productos sanos, según semana de gestación. Costa Rica, 1995-1996

\begin{tabular}{cccccccccccc}
\hline \multicolumn{2}{c}{ Sem 15 } & Sem 16 & Sem 17 & Sem 18 & Sem 19 & Sem 20 & Sem 21 & Sem 22 & Sem 23 & Sem 24 & Sem 25 \\
\hline n & 5 & 12 & 16 & 17 & 13 & 10 & 3 & 6 & 5 & 3 & 6 \\
\hline MM 2.5 & 1403.6 & 776.12 & 672.5 & 1365 & 2645 & 905 & 2037.5 & 3733.8 & 930 & 1097.5 & 2988.8 \\
Me & 1561.5 & 310.5 & 269 & 546 & 1058 & 362 & 815 & 1093.5 & 372 & 439 & 1195.5 \\
MM 0.25 & 140.4 & 77.6 & 67.2 & 136.5 & 264.5 & 90.5 & 203.8 & 373.4 & 93.0 & 109.8 & 298.9 \\
\hline
\end{tabular}

MM = Múltiplo de la mediana.

caso presentó niveles de AFP normal pero con un cariotipo que correspondió a un Síndrome de Turner.

\section{DISCUSIÓN.}

El diagnóstico cromosómico fetal mediante amniocentesis y cultivo de células fetales descamadas en el líquido amniótico, es el método más utilizado y forma parte de las normas de atención de la mujer embarazada de alto riesgo en la mayoría de los centros del mundo desarrollado (7). En algunos países de América Latina esta actividad se ha desarrollado casi exclusivamente en el ámbito privado y en los estratos sociales medio y alto (10). Costa Rica es una de las excepciones y la mayoría de las embarazadas del presente estudio, provienen de la seguridad social y de las clases media y baja.

Las formas más comunes de DTN son anencefalia con o sin espina bífida y espina bífida con o sin estar asociada a hidrocefalia. Para efectos del diagnóstico prenatal se debe hacer la distinción de defectos abiertos y cerrados. Un defecto abierto describe una situación en la cual hay comunicación entre el fluído cerebroespinal y el líquido amniótico, mientras que un defecto cerrado hay una piel gruesa que cubre el defecto. En general, cerca de la mitad de las malformaciones son anencefálicas y la mitad son espina bífidas y los defectos cerrados acontecen cerca del 10\% de todos los DTN (13).

La sangre fetal tiene un nivel normal de AFP en un DTN, la ruta por la que aumentan los niveles de AFP en el líquido amniótico se presume que puede ser por la salida del líquido cefaloraquídeo o por la trasudación o sangrado de capilares fetales directamente expuestos al fluído amniótico. Estas afirmaciones se apoyan en el hecho de que los niveles de AFP en espina bífida cerrada son normales o solo incrementados ligeramente (14).

El hallazgo de espina bífida se asocia a defectos cromosómicos en el 17\% de los casos y las anomalías más frecuentes son trisomía 18, trisomía 13, triploidía y translocaciones (8). Los hallazgos sonográficos más frecuentes de los fetos con trisomía 18, de 24 semanas gestacionales o menos, son: higroma quístico, engrosamiento de la nuca y mielomeningocele (8).

Se ha encontrado que niveles anormales tanto altos como bajos son predictivos de otras patologías adicionales, como embarazo molar, aborto retenido, prematuridad, muerte fetal, sobreestimación de la edad gestacional y más recientemente, síndrome de Down (15).

Entre las semanas 8 y 13 de gestación se dan cambios muy rápidos en los niveles de AFP en el líquido amniótico por lo que es muy difícil la interpretación clínica de esta medición y muy dependiente del cálculo de la edad gestacional de la paciente (16).

Los valores absolutos de las concentraciones de AFP ofrecen importantes variaciones regionales, de forma tal que los valores de la mediana deben establecerse en cada región o grupo poblacional (17). El error en la estimación de la edad gestacional es la primera causa de AFP elevada. Cuando se obtienen valores de AFP elevados se debe considerar:

1. Peso materno: mujeres más pesadas tienen niveles menores de dicha proteína, quizá como

\section{Revista Biomédica}




\section{Alfa-fetoproteína en el diagnóstico prenatal.}

consecuencia del efecto diluyente de un mayor volumen de sangre (18).

2. Raza: las mujeres de raza negra tienen niveles mayores que las blancas, orientales o de origen hispano (19).

3. Diabéticas: las mujeres diabéticas normalmente tienen niveles menores (20\%) que las no diabéticas (19).

4. Embarazo múltiple: los gemelos en promedio producen el doble de AFP que el feto único y mayores niveles de AFP sérica materna (19).

5. Ingesta de medicamentos: los niveles de AFP sérica materna disminuyen en las usuarias de alfametildopa y fenotiacinas.

Solamente un 2\% de las AFP elevadas están relacionadas con malformaciones fetales, fundamentalmente con defectos del tubo neural (20). Además una tercera parte de las pacientes con la primera muestra elevada tendrá un valor normal en la segunda determinación (21).

En el presente estudio obtuvimos niveles elevados en un caso de aberración cromosómica donde se esperarían niveles bajos, por lo que se debería repetir la determinación y revisar la edad gestacional de la madre ya que es una de las causas principales de falsos positivos.

Los valores de la concentración de AFP se expresan como múltiplos del valor medio, que se obtienen dividiendo cada resultado de la medición en el líquido amniótico de AFP entre la media calculada para la población de una determinada edad gestacional (13). La utilización del límite de normalidad en MM en vez de percentiles se debe a que el procedimiento presenta un cálculo más sencillo y más estable, dado que la proporción de gestaciones afectadas con niveles de AFP superiores a un múltiplo dado de mediana es poco probable que varíe de forma significativa entre centros o en el tiempo (17).

La AFP no es un marcador diagnóstico ni específico de los DTN u otras malformaciones congénitas, y su valor debe ser interpretado junto con los resultados de otros análisis bioquímicos en el suero materno, ultrasonido, etc., con el fin de optimizar el diagnóstico prenatal, disminuyendo así las posibilidades de falsos positivos y negativos.

La determinación de la AFP en el líquido amniótico es un método confiable para la detección de un posible DTN u otra anomalía cromosómica pero la amniocentesis conlleva un riesgo de daño fetal y no puede ser usada para un programa de tamizaje. Utilizando los marcadores anteriormente expuestos se puede seleccionar aquellos casos en los cuales es necesario realizarles una amniocentesis.

La meta es el diagnóstico de patología fetal lo más temprano en el embarazo para valorar la posibilidad de tratamiento intrauterino, interrupción del embarazo o preparación del núcleo familiar y del personal de salud, para la atención óptima del neonato afectado y así minimizar el daño y mejorar el tratamiento o rehabilitación. El diagnóstico prenatal de cromosomopatía permitió el asesoramiento genético y el manejo obstétrico y pediátrico de manera adecuada.

\section{AGRADECIMIIENTO.}

Este estudio fue financiado por el Organismo Internacional de Energía Atómica y la Vicerrectoría de Investigación de la Universidad de Costa Rica.

\section{REFERENCIAS}

1.- Milunsky A. Maternal serum screening for neural tube and other defects. In: Genetic disorders and the fetus. Diagnosis, prevention and treatment. 3d. Ed. Baltimore: Johns Hopkins; 1992. p. 507-63.

2.- Macri JN, Baker D, Baim R. Diagnosis of neural tube defects by evaluation of amniotic fluid. Clin Obstet Gynecol 1981;24:1089-01.

3.- Brock DJH. Prenatal diagnosis. Clinical methods. Br Med Bull 1976; 32: 16-20.

4.- Langman J. Embriología Médica. $3^{\text {a }}$ ed. México: Editorial Interamericana; 1976.

5.- Campbell RL, Dayton DH, Sohal GS. Neural tube defects: A review of human and animal studies on the etiology of neural tube defects. Teratology 1986; 34: 171-87.

6.- Cunningham FG, Gilstrap LC. Maternal serum alphafetoprotein screening. N Engl J Med 1991; 235: 55-6.

7.- Castro I, Escalante G, Mora H, Guerra D, Sánchez L, Peña 


\section{P Salas-Chaves, S Rodríguez-Aguilar, L Cunningham-Lucas, I Castro-Volio.}

C. Cariotipo de células fetales en el diagnóstico prenatal en Costa Rica. Rev Biol Trop 1995; 43: 31-7.

8.- Castro I, Sander K, Vargas M, Sánchez L, Escalante G. Cariotipos fetales en embarazos de alto riesgo genético provenientes de Hospitales de la Seguridad Social y de la Consulta Privada, de 1993 a 1998. Acta Med Cost 2000; 42: 25-30.

9.- Salas P, Rodríguez S, Villalobos A, Contreras P. Alfafetoproteína sérica materna: determinación de los valores medios normales en mujeres embarazadas costarricenses. Acta Med Cost 1999; 41: 28-33.

10.- Organización Panamericana de la Salud. Ejecución de las actividades de salud de genética en América Latina y el Caribe. Informe de la reunión de un grupo de expertos en genética médica. La Habana, Cuba; 9-11 de octubre, 1987.

11.- "Alpha-fetoprotein Immunometrics assay" Versión 3.2, London: Immunometrics Ltd, U.K.; July 1996.

12.- Piyasena RD, Lafranco G. Radioimmunoassay data proccessing program for IBM/PC computer. A user's manual. Vienna: International Atomic Energy Agency; 1989. p. 1154.

13.- Ruoslahti E, Tallberg T, Seppala M. Origin of proteins in amniotic fluid. Nature 1966; 212: 841-3.

14.- Simpson LJ. Maternal serum alpha-fetoprotein measurement in antenatal screening for anencephaly and spina bifida in early pregnancy. Lancet 1977; 1: 323.

15.- United Kingdom Collaborative Study: Maternal serum alpha-fetoprotein measurement in antenatal screening for anencephaly and spina bifida in early pregnancy. Lancet 1977; 1:323-32.

16.- Wathen N C. Alphafetoprotein levels in amniotic fluid from 8 to 18 weeks of pregnancy. Br J Obstet Gynecol 1993; 100:380-2.

17.- Schnittger A, Kjessler J. Alpha-fetoprotein screening in obstetric practice and the use of differential action limits. Acta Obstet Gynecol 1984; 17:16-41.

18.- Adams MJ, Windham, GC, James LM. Clinical interpretation of maternal serum alpha-fetoprotein concentrations. Am J Obstet Gynecol 1984; 148: 241-54.

19.- Macri NJ. Maternal serum alpha-fetoprotein screening, maternal weight and detection efficiency. Am J Obstet
Gynecol 1986; 155:758-60.

20.- Moína MJ, Cárdenas M, Agramunt G, Venta R, Alvarez FV. Utilidad de la alfafetoproteina sérica materna como parámetro de riesgo del embarazo. Prog Obstet Ginecol 2001; 44:252-60.

21.- Verpyck E, Degree S, Hellot MF, Descargues G, Phillippe C, Labadie G. Amniotic fluid alpha-fetoprotein is not a useful biological marker of pregnancy outcome. Prenat Diag 1999; 19:1031-4.

\section{Revista Biomédica}

